\title{
Transpositional immunity: immunotherapeutical approach against bovine leukemia virus
}

\author{
RA Roa-Castellanos
}

\author{
Address: Faculty of veterinary medicine, La Salle University, Instituto de Bioetica, Pontificia Universidad Javeriana, Bogota DC, Colombian \\ National Health Instiute, Colombian National Insitute of Cancerology, Colombia \\ from Frontiers of Retrovirology: Complex retroviruses, retroelements and their hosts \\ Montpellier, France. 21-23 September 2009 \\ Published: 24 September 2009
}

Retrovirology 2009, 6(Suppl 2):P78 doi:10.1 186/1742-4690-6-S2-P78

This abstract is available from: http://www.retrovirology.com/content/6/S2/P78

(c) 2009 Roa-Castellanos; licensee BioMed Central Ltd.

Bovine Leukemia Virus, an unparalleled oncogenic retrovirus related taxonomically to HTLV and HIV, was diagnosed using Agar Gel Immunodiffusion (AGID) among 485 Holstein cows older than two years in five herds across the northwest of the Bogotá valley. Real serological prevalence of BLV infection varied from $68 \%$ to $89 \%$, finding similar frequencies to those encountered in dairy regions of the USA and Canada. The leukemic condition of B1 cells (CD5+) called Persistent Lymphocytosis (PL) was confirmed by carrying out White Blood Cells Counts (WBC) in seropositive animals for three months after taking initial samples. 56 seropositive individuals (11.55\%) exhibited notorious PL. Only 51 animals were eventually selected to from the group to be evaluated. The group of 51 animals was divided into two subgroups. Subgroup 1, which comprised 11 control animals, did not receive any treatment. The second group, 40 experimental cows, was given three types of Th1/Th3-immune response-stimulant, previously selected during the first phase of the investigation. $78 \%$ of the experimental group developed slight hyperthermia $\left(40^{\circ} \mathrm{C}+/-1^{\circ} \mathrm{C}\right)$. These animals obtained normalization of their blood values, as far as 75 days after-treatment when the experiment was over. In contrast, those experimental cows that did not show any thermic increase or that did not receive complete treatment, in spite of recovering temporarily, returned to the leukemic status. A case of incipient retroorbitary lymphosarcoma, confirmed by histopathology, was submitted to therapy. The growth rate of this tumor decreased substantially in comparison with two untreated cases suffering from comparable presentations; at the extent that one year after therapy the tumor maintained practically the same size. These results suggest that 1) Antagonistic cytokine dynamics (e.g.Th2 cellular system predominance over Th1 and Th3 system) involved in pathogenesis of these retroviral presentations, as well as their initial forms of neoplasia, may be reversed to protract expectancy of life in afflicted patients. 2) Interestingly, different manifestations of BL caused by BLV such as asymptomatic stadium, $\mathrm{PL}$, and Lymphosarcoma - also seen in other retroviral infections in different species - seem to be consecutive stages along an only process. Considering the foregoing, the length of each presentation would be chiefly determined by means of inherited MHC dominance and its subproducts (Interleukins, chemokines, Inmunoglobulins, cell receptors involved, etc.) in every individual response against retroviruses. 3) Extracorporeal systems of hyperthermia might not exert the same response on viral reservoirs as this procedure does through Heat Shock Proteins (HSP) molecules and Th1/Th3 exacerbation. To generate an effective response against this imperceptible agent (BLV), which is using a Trojan horse-like mechanism to go unnoticed before the immune system; it is necessary to stimulate NK and $\gamma \delta$ cells via IFN- $\gamma / \alpha$, IL- 1, IL- 2 , IL-12, IL-15, TNF- $\alpha$, MHC I/MHC III, Cortisol and HSPs. 4) This type of therapy, called as Transpositional Immunity, would strengthen epidemiological strategies to fight BLV infection; moreover, it could be seen as an animal model for similar human and non-ruminant illnesses. Resembling Hairy Cell Leukemia and Chronic Lymphocytic Leukemia in humans, including its Ritcher's syndrome, and being recently associated with Human Breast Cancer in addition to Lymphoma in chimpanzees fed on BLV infected milk; BLV cannot be ruled out as a carcinogen zoonotic agent yet. 